\title{
Fenómeno de doble cápsula bilateral en mamoplastia de aumento
}

\section{Double bilateral capsule in mammoplasty of increase}

\author{
Francisco Javier Velázquez Aranda, ${ }^{*}$ Francisco Javier Soto Villanueva, ${ }^{\ddagger}$ \\ Carlos Fernando Nicolás Cruz, ${ }^{\ddagger}$ Martín Manzo Hernández*
}

Citar como: Velázquez AFJ, Soto VFJ, Nicolás CCF, Manzo HM. Fenómeno de doble cápsula bilateral en mamoplastia de aumento. An Med (Mex). 2020; 65 (4): 315-319. https://dx.doi.org/10.35366/97471

\section{RESUMEN}

La mamoplastia con la utilización de implantes mamarios es un procedimiento cada vez más frecuente, tanto en cirugía estética para mamoplastia de aumento como para reconstrucción oncológica después de mastectomía total. El aumento en la realización de este procedimiento ha traído consigo un incremento proporcional de las complicaciones postquirúrgicas asociadas a éste. Aunque las complicaciones asociadas con la mastoplastia con colocación de implantes son diversas, el fenómeno de doble cápsula es una complicación particularmente infrecuente, la cual en los últimos años ha cobrado un interés importante dentro de la cirugía plástica. Una búsqueda intencionada en las principales bases de datos médicas confirma lo inusual del caso, al encontrar no más de 40 artículos publicados. En el presente artículo presentamos el caso del hallazgo de fenómeno de doble cápsula bilateral en una paciente con implantes mamarios texturizados (BIOCELL ${ }^{\mathrm{MT}}$ McGhan ST110) de localización retromuscular con estado postquirúrgico de ocho años, en una mujer de 46 años aparentemente sana. En él se observa una doble cápsula fibrosa, una íntimamente adherida al implante y otra más holgada recubriendo ambas estructuras. Así como un análisis de la literatura que ha sido publicada en los últimos años, haciendo enfoque en la fisiopatología y las teorías aceptadas en la etiología de este fenómeno.

Palabras clave: Contractura capsular, doble cápsula, implantes mamarios texturizados.

Nivel de evidencia: IV

\begin{abstract}
Mammoplasty with the use of breast implants is an increasingly common procedure, both in cosmetic surgery for augmentation mammoplasty and for subsequent oncological reconstruction of a total mastectomy. The increase in the performance of this procedure has been betrayed by a proportional increase in postsurgical complications at this very moment. Although complications associated with implants mastoplasty are diverse, the phenomenon of double capsule is a complication infrequent, in recent years has gained an important interest in plastic surgery. An intentional search in the main medical databases confirms the unusualness of the case, finding no more than 40 articles published. In the present article we present the case of the finding of a bilateral double capsule phenomenon in a patient with textured mammary implants (BIOCELL $L^{M T}$ McGhan ST-110) of retromuscular location with an 8-year postoperative state, in a apparently healthy 46-year-old woman. It shows a double fibrous capsule, one closely attached to the implant and another looser covering both structures. As well as an analysis of the literature recently published in recent years, focusing on the physiopathology and accepted theories in the etiology of this phenomenon.
\end{abstract}

Keywords: Capsular contracture, double capsule, textured breast implants.

Level of evidence: $I V$

\footnotetext{
* Médico Cirujano Plástico y Reconstructivo. Miembro de la Asociación Médica. Centro Médico ABC.

‡ Médico Residente de Cirugía General.
}

\author{
Correspondencia: Carlos Fernando Nicolás Cruz \\ Centro Médico ABC Campus Santa Fe. \\ Av. Carlos Fernández Graef Núm. 154, Col. Tlaxala, 05300, \\ Cuajimalpa de Morelos, Ciudad de México, México. \\ Tel: 55 2699-5017 \\ E-mail: nicolascarlosf12@gmail.com
}




\section{INTRODUCCIÓN}

La colocación de implantes mamarios como opción terapéutica al aumento en el tamaño de las glándulas mamarias es un procedimiento quirúrgico cada vez más en aumento. Aproximadamente 1.5 millones de mujeres se realiza mamoplastia de aumento con implantes mamarios. ${ }^{1}$ Casi todos los dispositivos implantables sintéticos, incluidos los implantes mamarios, formarán en mayor o menor medida un grado variable de encapsulación de la cicatriz compuesta por colágeno y fibroblastos desorganizados. La observación de cápsulas dobles se ha descrito en series limitadas y los informes de casos predominan en implantes texturizados, específicamente con BIOCELL. ${ }^{2}$

El fenómeno de doble cápsula se refiere al hallazgo de dos láminas separadas que rodean al implante mamario. La cápsula interna está adherida al implante protésico, mientras que la otra cápsula se encuentra rodeando el tejido celular subcutáneo o glandular. ${ }^{3}$

Se ha observado que, con el paso del tiempo, el riesgo de desarrollar seromas tardíos, infecciones y en menor frecuencia metaplasia sinovial requiere revisiones quirúrgicas constantes. La contractura capsular es la causa más frecuente de reintervención, mientras que la asimetría, que es la segunda causa, es secundaria a la rotación o al desplazamiento del implante. ${ }^{4,5}$ La patogénesis de la contractura capsular tiene diversas variantes, se ha postulado que es a partir del proceso de inflamación crónico propiciado por fenómeno de cuerpo extraño del implante protésico, a esto se añaden potenciadores y factores clave como modificación del biofilm y la contaminación bacteriana. ${ }^{6}$ En esta investigación reportamos el caso de una mujer de 46 años que, secundario a la contractura capsular de ambos implantes mamarios, es sometida a revisión quirúrgica y es encontrado como hallazgo incidental la presencia de doble cápsula; asimismo, hacemos una revisión de la literatura, con artículos publicados en los últimos 10 años en revistas de alto y mediano impacto.

\section{REPORTE DE CASO}

Presentamos el caso de una mujer de 46 años, refiere como único antecedente de importancia fragilidad capilar, que se presenta con equimosis ante mínimo trauma, tabaquismo positivo a razón de cinco cigarrillos al día en los últimos 10 años. Como antecedentes quirúrgicos, tres cesáreas sin complicaciones, mastopexia con implantes a la edad de 36 años, con colocación de implantes redondos simétricos de $230 \mathrm{~g}$ en plano submuscular, texturizados, McGhan ST-110 y a la edad de 44 años resección de tumoración benigna en seno izquierdo, reportada como fibroadenoma mamario.

Acude a consulta para valoración médica por presencia de dolor en ambas mamas y ptosis mamaria bilateral; se decide plan de capsulotomía más cambio de implantes con mastopexia mamaria, además de complementar con abdominoplastia y liposucción de flancos en segundo tiempo quirúrgico. Durante el procedimiento se realiza abordaje por cicatriz de pexia previa, identificando cápsula, incidiendo y observando la presencia del implante cubierto por segunda cápsula, el cual se encontraba libre dentro de dicho bolsillo (Figura 1). Se extrae implante con cápsula íntegra y se realiza capsulotomía anterior únicamente, se deja porción posterior de la misma. Se aborda mama izquierda y observamos el mismo fenómeno. Al abrir la cápsula interna que contiene los implantes, encontramos implantes descritos en párrafo anterior sin datos de ruptura o exudado en su interior (Figura 2). Se procede a continuar con plan preestablecido y se colocan implantes de $220 \mathrm{~g}$ redondos texturizados de perfil alto marca Allergan TSF N-TSF, además de realizar el resto de los procedimientos programados, llevados a cabo de manera rutinaria y sin ninguna complicación o hallazgo distinto. La recuperación del caso se desarrolló de forma satisfactoria en las semanas posteriores al procedimiento quirúrgico.

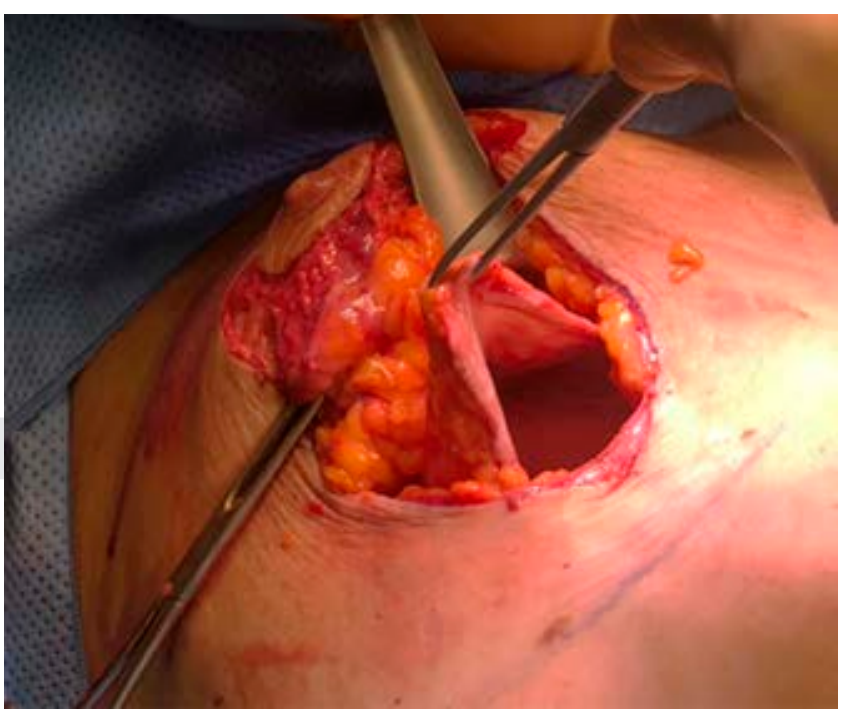

Figura 1: Se realiza abordaje quirúrgico en pexia previa en mama derecha, donde se observa primera cápsula adherida al tejido celular subcutáneo y el espacio formado entre ésta y la cápsula adherida al implante mamario. 

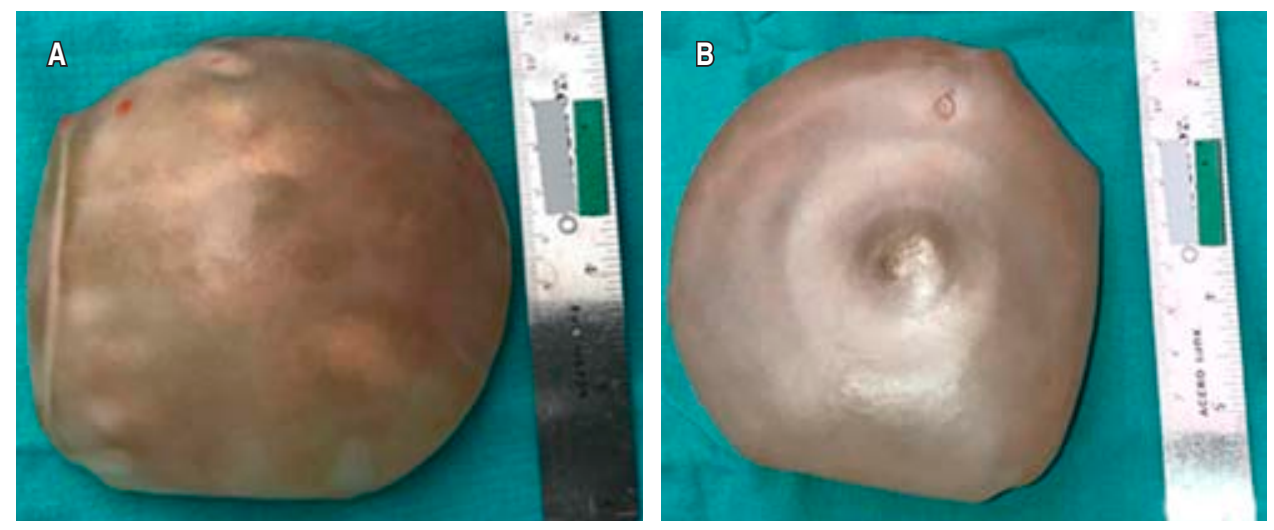

Figura 2:

Se observa implante mamario derecho recubierto con la cápsula adherida, íntegra y sin puntos de fijación. A) Vista de su cara anterior. B) Vista de su cara posterior.

Se envían muestras a estudio histopatológico, reportándose cápsulas mamarias con inflamación crónica inespecífica leve y calcificación distrófica, constituidas por material eosinofílico amorfo y fibroblastos, en algunos sitios laminado, sin evidencia de malignidad.

\section{DISCUSIÓN}

Los implantes mamarios se encuentran entre los tipos más utilizados de implantes permanentes en medicina moderna y tienen aplicaciones tanto estéticas como reconstructivas. La biocompatibilidad de éstos se considera excelente y ha sido estudiada por más de 50 años. Aun así, numerosos factores afectan la bioestabilidad de la cápsula-implante a lo largo del tiempo. ${ }^{2}$

Resulta interesante que, hasta el momento, en bases de datos y buscadores médicos importantes como «PubMed», «Ovid», «MEDLINE», al buscar de manera dirigida, se encuentran no más de 40 artículos publicados sin discriminar entre el tipo de estudio, la nacionalidad o el idioma. Por ello, consideramos importante el reporte del fenómeno poco usual en la formación de doble cápsula.

Todos los implantes mamarios producen una respuesta granulomatosa que finaliza con una cápsula fibrosa con mínima vascularidad, con predominio en su periferia de macrófagos y fibroblastos.

En fases iniciales de la cicatrización, ocurre la formación de un seroma que ocurre en menor medida en los implantes lisos, pero que puede llegar a ser considerable en los implantes texturizados, como los utilizados por la paciente del caso (BIOCELL McGhan ST-110). Los fabricantes argumentan que el efecto rugoso de la superficie promueve mayor adhesión a tejidos, rápida fijación y reducción del riesgo de contractura capsular. Sin embargo, también presenta más riesgo de producción de seromas y mayor frecuencia en la formación de doble cápsula, la cual ha sido reportada en diversas revisiones. ${ }^{7,8} \mathrm{~A}$ esto se añade la reciente asociación con el linfoma anaplásico de células gigantes, se han reportado hasta ahora 600 casos en todo el mundo, los cuales tienen como características la aparición a 10 años del procedimiento de colocación y la probable etiología de contaminación del implante y contracción capsular, lo que propicia un entorno inflamatorio al implante. . $^{9,10}$

Basándose en literatura reciente, se han establecido cuatro teorías para la formación de dobles cápsulas en los implantes mamarios: la primera hipótesis se basa en el movimiento de la prótesis dentro de un bolsillo de tejido de gran tamaño; los macro y micro movimientos del implante evitan la adhesión de la superficie texturizada del implante a los tejidos circundantes. ${ }^{11} \mathrm{La}$ segunda hipótesis sugiere una etiología mecánica mediante la cual el esfuerzo de cizallamiento aplicado al complejo de la cápsula protésica aleja la prótesis de la cápsula; esta separación conduce a la creación posterior de una nueva capa interna de cápsula en contacto directo con la prótesis. Según lo propuesto por Hall-Findlay, la fricción continua entre la cubierta texturizada del implante y la cápsula original conduce a una acumulación de líquido similar a seroma; la aparición secundaria de células derivadas de este fluido sobre la superficie del implante inicia el desarrollo de una nueva capa interna de cápsula adherente. ${ }^{12}$ La base de la tercera hipótesis es que se forma un seroma de etiología variable alrededor de la prótesis, que después conduce al desarrollo de una nueva cápsula interna, lo cual es el origen del exudado seroso infeccioso, alérgico o hemorrágico. Los organismos encontrados con mayor frecuencia, en caso de ser infecciosa, han sido S. epidermidis y $S$. aureus. ${ }^{13,14}$ La cuarta hipótesis tiene una base 
mecánica y propone que las fuerzas de cizallamiento causan el desprendimiento del complejo de la cápsula del implante del tejido mamario circundante, dejando así la cápsula original en bloque con el implante texturizado. Después se desarrolla una nueva capa de cápsula externa para producir el fenómeno de doble cápsula. ${ }^{2}$

Se sospecha que estos fenómenos ocurren debido a la estructura porosa del implante BIOCELL, el cual presenta a nivel microscópico un alto grado de angulaciones y pequeños radios de curvatura en los bordes, haciendo más comunes la formación de células gigantes multinucleadas y la formación de seroma, así como mayor frecuencia en la formación de doble cápsula. ${ }^{15}$

En el caso mencionado (Figura 3), el implante mamario de BIOCELL McGhan ST-110 tuvo una colocación submuscular, la literatura menciona que son precisamente ese tipo de implantes y materiales rugosos los que generan mayor reacción inflamatoria, así como mayores complicaciones cuando se colocan en el plano retromuscular, así como una reacción menor en tejido adiposo. ${ }^{16,17}$

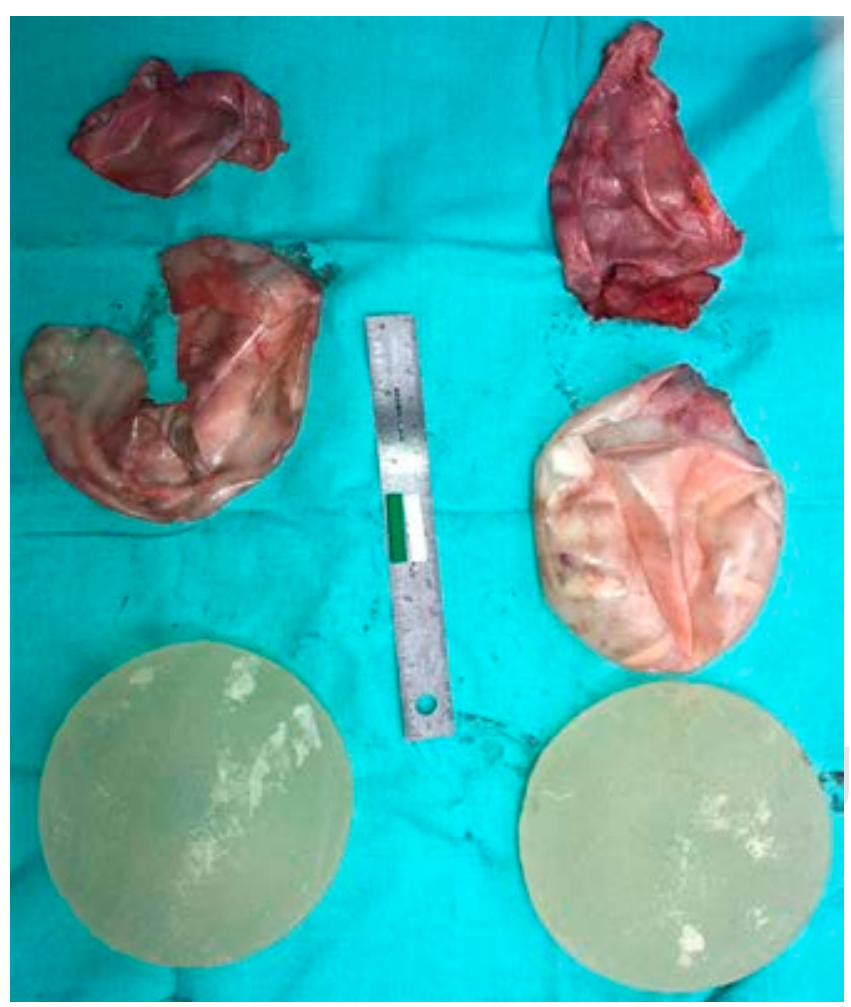

Figura 3: Fotografía tomada al final del evento quirúrgico, se presentan los implantes mamarios texturizados, la cápsula formada y que previamente estaba adherida a éstos, así como la segunda cápsula que se formó adhiriéndose al tejido celular subcutáneo.

\section{CONCLUSIÓN}

En la actualidad, se desconoce con certeza la incidencia del fenómeno de doble cápsula en la mamoplastia de aumento. De igual forma, la etiología de este fenómeno aún no está totalmente clara; sin embargo, la teoría mecánica del origen de la doble cápsula parece ser la causa más probable de este fenómeno. Se debe continuar realizando una constante investigación, con reportes de serie de casos y metaanálisis, en búsqueda de un implante ideal, éste debería ser uno que no se degrade, que tenga un beneficio biológico, que produzca un grado limitado de respuesta inflamatoria y reduzca la generación, alineación y orientación de las fibras de colágeno, que propician la aparición de esta entidad. Como cirujanos plásticos, debemos tener un conocimiento amplio sobre los tipos y características macro y microscópicas de los implantes, así como de sus beneficios y complicaciones particulares, ya que las variables en la aparición de complicaciones como contracturas capsulares, aparición de seromas tardío y tempranos, así como de doble cápsula pueden ser atribuidos al cirujano, la sensibilidad del paciente al implante, el sitio del implante y el tipo de implante colocado.

\section{BIBLIOGRAFÍA}

1. ISAPS Global Statistics 2016. July 1, 2016. 2. U.S. Food and Drug Administration. Disponible en: www.isaps.org/Media/ Default/global-statistics/2016\%20ISAPS\%20Results.pdf.

2. Hall-Findlay EJ. Breast implant complication review: double capsules and late seromas. Plast Reconstr Surg. 2011; 127 (1): 56-66.

3. Maxwell GP, Brown MH, Oefelein MG, Kaplan HM, Hedén P. Clinical considerations regarding the risks and benefits of textured surface implants and double capsule. Plast Reconstr Surg. 2011; 128 (2): 593-595.

4. Update on the Safety of Silicone Gel-filled Breast Implants. Center for Devices and Radiological Health \& U.S. Food and Drug Administration, FDA, 2011.

5. Caplin DA, Vargo JM, Canady J, Hammond D. Long-term clinical performance of memoryshape silicone breast implants in breast augmentation: prospective data through 9 years. Plast Reconstr Surg. 2014; 134 (4): 92-93.

6. Wan D, Rohrich RJ. Revisiting the management of capsular contracture in breast augmentation: a systematic review. Plast Reconstr Surg. 2016; 137 (3): 826-841.

7. Matteucci P, Fourie le R. Double capsules related to dynamic malrotation of breast implants: a causal link? Br J Plast Surg. 2004; 57 (3): 289.

8. Nava MB, Rancati A, Angrigiani C, Catanuto G, Rocco N. How to prevent complications in breast augmentation. Gland Surg. 2017; 6 (2): 210-217.

9. Miranda RN, Aladily TN, Prince HM, Kanagal-Shamanna R, de Jong D, Fayad LE et al. Breast implant-associated anaplastic large-cell lymphoma: long-term follow-up of 60 patients. J Clin Oncol. 2014; 32 (2): 114-120. 
10. Walker JN, Pinkner CL, Pinkner JS, Hultgren SJ, Myckatyn TM. The detection of bacteria and matrix proteins on clinically benign and pathologic implants. Plast Reconstr Surg Glob Open. 2019; 7 (2): e2037.

11. Góes JC, Landecker A. Optimizing outcomes in breast augmentation: seven years of experience with the subfascial plane. Aesthetic Plast Surg. 2003; 27 (3): 178-184.

12. Toscani M. Breast implant complication: calcifications in the double capsule. Plast Reconstr Surg. 2013; 131 (3): 462-464.

13. Pinchuk V, Tymofii O. Seroma as a late complication after breast augmentation. Aesthetic Plast Surg. 2011; 35 (3): 303-314.

14. Allan JM, Jacombs AS, Hu H, Merten SL, Deva AK. Detection of bacterial biofilm in double capsule surrounding mammary implants: findings in human and porcine breast augmentation. Plast Reconstr Surg. 2012; 129 (3): 578-580.

15. Picha GJ. Mammary implants: surface modifications and the soft tissue response. Semin Plast Surg. 1991; 5 (2): 54-79.

16. Atlan M, Bigerelle M, Larreta-garde V, Hindié M, Hedén P. Characterization of breast implant surfaces, shapes, and biomechanics: a comparison of high cohesive anatomically shaped textured silicone, breast implants from three different manufacturers. Aesthetic Plast Surg. 2016; 40 (1): 89-97.

17. Smahel J, Hurwitz PJ, Hurwitz N. Soft tissue response to textured silicone implants in an animal experiment. Plast Reconstr Surg. 1993; 92 (3): 474-479. 